\title{
Latitudinal Variations of Line-of-Sight Velocity Oscillations in the Photosphere, Chromosphere and Prominences
}

\author{
G.P. Mashnich and V.S. Bashkirtsev \\ Institute of Solar-Terrestrial Physics, Irkutsk, P.O. Box 4026, Russia
}

\begin{abstract}
By studying line-of-sight velocity time variations in prominences, we found that velocity oscillations with a period over $40 \mathrm{~min}$ have a reasonably well-marked dependence of the period length with heliolatitude. Simultaneous observations of line-of-sight velocities in the photosphere and chromosphere showed that quasi-hourly oscillation periods at these levels in the solar atmosphere and in prominences have a similar latitudinal behavior.
\end{abstract}

\section{Introduction}

While speculating concerning the origin of oscillations in prominences, we tried to identify some peculiarities of oscillation parameters depending on the phase of solar activity cycle, and on the position of prominences relative to zones of activity. As a result, the study revealed a reasonably clear-cut heliolatitudinal dependence of the value of the line-of-sight velocity oscillation period in prominences (Bashkirtsev and Mashnich 1993). Line-of-sight velocity observations in prominences covering the period 1981-1991 were used in our investigation.

Whenever the issue concerns fundamental problems of the solar interior and differential rotation, of significant importance are reliable experimental data on latitudinal variations of physical plasma parameters, the geometrical size of solar features, and on their behaviour within the course of the evolution of a solar cycle. Directly or indirectly, observational data make it possible to get closer to realistic models. By investigating the line-of-sight velocity time variations in prominences, we defined the oscillations with periods over 40 minutes as the forced ones and supposed that the source of such oscillations resides in layers of the solar atmosphere below the prominence. If this is so, then the latitudinal peculiarities in quasi-hourly oscillations must be revealed, at least in the visible layers of the solar atmosphere. We present results derived by investigating the heliolatitude dependence of oscillation parameters in the photosphere and chromosphere.

\section{Observations}

The observations were made at the Sayan-Mountain Solar Observatory during August-September 1995 and in July 1996 at the horizontal solar telescope. Velocity variations were measured using a high-precision differential method of measuring line-of-sight velocities which has been used with the solar magneto- 
graph. The concept of the instrument for differential measurements of the lineof-sight velocity and its implementation were detailed in a paper of Kobanov (1983). Using an additional photomultiplier we carried out measurements simultaneously in the photosphere $(\lambda 485.97 \mathrm{~nm})$ and the chromosphere $(\lambda 486.13$ $\mathrm{nm}$ ), with the $2 " \times 8$ " entrance slit and with the spectrograph dispersion in $\mathrm{V}$ of the order of $0.32 \AA / \mathrm{mm}$. The solar image was kept on the spectrograph slit by means of the photoelectric guider; solar rotation was automatically compensated. With allowance made for distorting factors, line-of-sight velocity measurements were made to an accuracy no worse than $20 \mathrm{~m} \mathrm{~s}^{-1}$. Areas to be investigated were chosen in the light reflected from the spectrograph mirror slit and passed through a Halle $\mathrm{H} \alpha$ filter. It is known that short-period oscillations (of about $3.7 \mu \mathrm{Hz}$ ) are vertical ones; therefore, the amplitude of such oscillations decreases from the center to the solar limb. The behaviour of the quasi-hourly oscillations of the chromosphere is the reverse (Bashkirtsev and Mashnich 1987): at the center of the solar disk they are virtually nonexistent, and with increasing distance from the center to the limb their amplitude increases. Taking these factors into account, we chose, for the observation, regions of the quiet Sun in longitude about $40^{\circ}$ near the latitudes $0^{\circ} 25^{\circ} 50^{\circ}$ and $65^{\circ}$. It is at these latitudes that we detected deviations from the mean values of the line-of-sight velocity, quasi-hourly oscillation period in prominences. The length of one recording was typically not less than 120 minutes; hence, during the daytime we were able to take one recording for each of the latitudes, provided that weather conditions allowed. Such a recording length gives sufficient resolution in the spectrum. The accuracy of determining the period, $P$, as shown by Djurovich and Pâquet (1994), is no worse than $P^{2} / 2 T$, where $T$ is the length of a time period. For example, when $T=2 P$, the error in determining the period does not exceed $P / 4$. Time sequences were pretreated; after that, the method of correloperiodogram analysis was used to calculate the line-of-sight velocity oscillation spectra for the frequency region less than $1 \mu \mathrm{Hz}$. Of the spectra obtained, we picked components with the largest amplitude, at a significance level $\geq 0.99$, whose values were plotted on the "period-latitude" diagram.

Proceeding in this way, we obtained heliolatitudinal dependences of the value of the quasi-hourly oscillation period. Figure 1 shows the latitudinal variations of the value of the period for the photosphere and Figure 2 for the chromosphere. For comparison, Figure 3 shows the latitudinal variations for the photosphere (dashed line), the chromosphere (dash-dotted line), and prominences (solid line). Even with small statistics, all dependences reveal deviations from the mean value of the period to maximum values at latitudes about $25^{\circ}$, and to minimum values at latitudes $45^{\circ}-50^{\circ}$.

\section{Discussion}

The quasi-hourly oscillations observed in prominences are interpreted in some papers (Balthasar et al. 1988) as eigen-oscillations caused by the geometry and physical conditions of the prominence itself. However, the coincidence of the latitudial dependences of the oscillation period at different levels of the solar atmosphere, as is evident from Figure 3, permit the quasi-hourly oscillations in prominences to be treated as forced ones. The photosphere, the chromosphere 


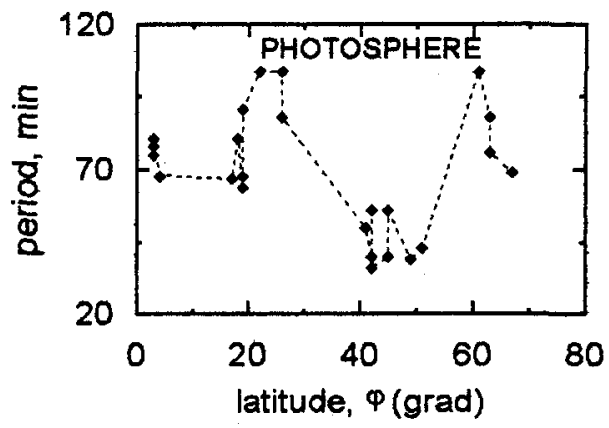

Figure 1. Latitudinal variations of the quasi-hourly oscillation period for the photosphere.

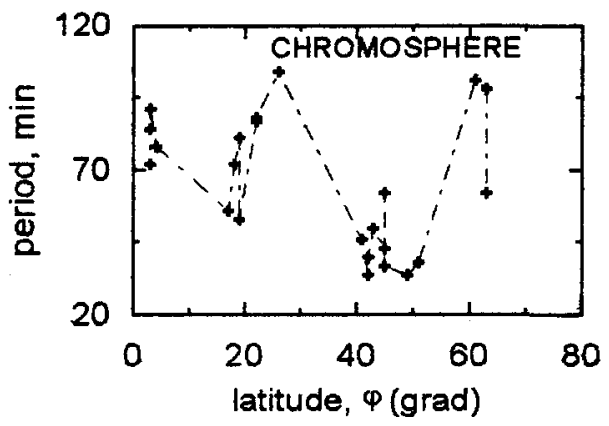

Figure 2. Latitudinal variations of the quasi-hourly oscillation period for the chromosphere.

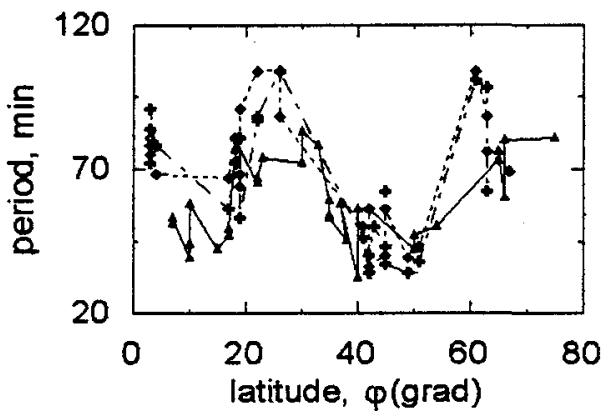

Figure 3. Comparison of the latitudinal variations of the oscillations in the photosphere, chromosphere and prominences.

and the prominence should be regarded as a unified oscillatory system for 
frequencies less than $1 \mu \mathrm{Hz}$.

It is not our intent here to provide an explanation for the presence of maxima and minima of the quasi-hourly oscillation period at certain latitudes, because of the small amount of observational data. It should be noted that some investigations detected variations of different physical parameters of solar plasma with latitude. A few examples are given below.

It is known that the solar magnetic field in the corona has a latitudinal zonal structure. Using data on the structure of the coronal magnetic field, Makarov $(1983,1984)$ showed that at maximum activity each solar hemisphere exhibits two global magnetic neutral lines at latitudes $20^{\circ}$ and $40^{\circ}$ and one line near the equator. In a series of papers addressing the observed frequency splitting of the p-modes, variations in the internal angular rotation velocity were calculated depending on the latitude and solar radius. Rhodes et al. (1990) carried out a comparison of the variations in inner angular rotation velocity depending on latitude and solar radius from observational data obtained at different observatories. A good agreement of results was found, and it was shown that the variations in inner angular velocity for $R=(0.6-0.95) R$ of the Sun behave differently for the equatorial, middle and near-polar latitudes. Rimnele and Schroter (1989), based on maps of line-of-sight velocities of the photosphere on the solar limb, found that the mean size of supergranulation cells varies with heliolatitude, showing a statistically significant minimum at the middle latitudes.

Our obtained result on the latitudinal variations of the value of the oscillation period in the photosphere, chromosphere and prominences is important not only for understanding the origin of prominences but, along with the above mentioned experimental data, is of interest when solving experimental problems of differential rotation and the solar interior.

Acknowledgments. We are indebted to A.V. Mordvinov for help with a mathematical treatment of the data and to N.I. Kobanov for help in preparing the instrument in readiness for the observation. This work was done with support of the Russian Foundation for Fundamental Research under grants 960216647, 970226726, and Governmental Support Grant for Leading Scientific Schools 961596733 .

\section{References}

Balthasar, M., Stellmacher, G. and Wiehr, E. 1988, A\&A, 204, 286

Bashkirtsev, V.S. and Mashnich, G.P. 1987, Sol. Phys., 109, 399

Bashkirtsev, V.S. and Mashnich, G.P. 1993, A\&A, 279, 610

Djurovich, D. and Pâquet, P. 1994, Sol. Phys., 152, 497

Kobanov, N.I. 1983, Sol. Phys., 82, 237

Makarov, V.I. 1983, Solnechnye Dannye, No.10, 93

Makarov, V.I. 1984, Solnechnye Dannye, No. 9, 52

Rhodes, E., Cassiani, A., Korzenik, S., Tomczyk S., Ulrich R. and Wooard, M. $1990, \mathrm{AJ}, 351,687$

Rimnele, T. and Schroter, E.H. 1989, A\&A, 221, 137 